\title{
Decreased expression of microRNA-145 promotes the biological functions of fibroblasts in hypertrophic scar tissues by upregulating the expression of transcription factor SOX-9
}

\author{
SHOUJIE WANG ${ }^{1}$, CAIYUN LI $^{1}$, YIJIA YU ${ }^{1}$ and JIANJUN QIAO ${ }^{2}$ \\ Departments of ${ }^{1}$ Plastic Surgery and ${ }^{2}$ Dermatology, The First Affiliated Hospital, \\ Zhejiang University, Hangzhou, Zhejiang 310003, P.R. China
}

Recieved July 23, 2017; Accepted December 8, 2017

DOI: $10.3892 /$ etm.2019.7972

\begin{abstract}
The present study aimed to determine the expression of microRNA (miRNA or miR)-145 in hypertrophic scars at the tissue and cellular levels, and to investigate its biological functions and mechanism of action. A total of 36 patients who were diagnosed with hypertrophic scar were included in the present study. Reverse transcription-quantitative polymerase chain reaction was used to determine the expression of miR-145 in tissues and fibroblasts. Primary fibroblasts were transfected with negative control miRNA, miR-145 mimics or inhibitor. A Cell Counting Kit-8 assay was performed to determine the level of proliferation of fibroblasts. Flow cytometry was employed for cell cycles determination and apoptosis in fibroblasts. A Matrigel assay was used to evaluate the invasion ability of fibroblasts. Western blotting was used to determine the expression of the transcription factor SOX-9 (SOX-9) protein in fibroblasts. Rescue experiments were performed to examine the effect of SOX-9 on the regulation of fibroblasts by miR-145. The dual luciferase reporter assay was performed to identify the direct interaction between SOX-9 and miR-145. The expression of miR-145 was reduced in hypertrophic tissues and fibroblasts. Overexpression of miR-145 inhibited the proliferation, G1/S phase transition and invasion of fibroblasts, and promoted the apoptosis of fibroblasts. In addition, overexpression of miR-145 inhibited SOX-9 protein expression. By contrast, the expression of SOX-9 reversed the effects of miR-145 on the proliferation, cell cycle, apoptosis and invasion of fibroblasts. The miR-145 seed region was able to bind with the 3'-untranslated region of the SOX-9 mRNA to regulate its expression. The present study demonstrated that miR-145 expression is reduced in hypertrophic scar tissues
\end{abstract}

Correspondence to: Dr Jianjun Qiao, Department of Dermatology, The First Affiliated Hospital, Zhejiang University, 79 Qingchun Road, Hangzhou, Zhejiang 310003, P.R. China

E-mail: qiaoderm@163.com

Key words: microRNA-145, transcription factor SOX-9, fibroblasts, hypertrophic scar and negatively associated with SOX-9 expression. In addition, miR-145 inhibits the proliferation, cell cycle and invasion, and promotes the apoptosis of fibroblasts by down-regulating the expression of SOX-9. The current study provides a potential target for the clinical diagnosis and treatment of hypertrophic scars.

\section{Introduction}

A hypertrophic scar is a clinically common skin fibroplasia disease that is caused by an excessive healing response of the human body to trauma (1). It is characterized by the abnormal proliferation of fibroblasts, abundant collagen synthesis and excessive collagen deposition (2). It was recently reported that the incidence of hypertrophic scars in burn patients in China reached $90 \%$ (3). Scar formation affects a patient's appearance, and results in contracture that may lead to different degrees of dysfunctions including tendon contracture, joint dislocation and dyskinesia (4,5). At present, the pathogenesis of hypertrophic scar has not been fully elucidated. It is widely accepted that fibroblasts are the major effector cells in hypertrophic scars, and abnormal cell proliferation and extracellular matrix deposition are the main characteristics of hypertrophic scars (6). The transforming growth factor (TGF)-GFmothers against decapentaplegic homolog (SMAD) signaling pathway is closely associated with a variety of physiological and pathological processes including collagen metabolism and the proliferation, differentiation, migration and apoptosis of fibroblasts $(7,8)$. Therefore, studies on the TGF- $\beta$ /SMAD signaling pathway in fibroblasts are important to elucidating the mechanisms of formation and development in hypertrophic scars.

microRNAs (miRNAs or miRs) form a class of non-encoding small RNA molecules with 18-24 nucleotides. miRNA molecules bind with the 3'-untranslated region (UTR) of mRNA to inhibit its translation into proteins, and are associated with the regulation of pathophysiological processes including cell proliferation, migration, apoptosis, autophagy, differentiation and tumor formation $(9,10)$. It has been reported that miRNAs regulate the formation and repair of skin and its accessory organs, suggesting that miRNAs serve important roles in hypertrophic scar (11). In addition, the abnormal expression of miRNA molecules was identified in 
hypertrophic scar tissues, suggesting that miRNA molecules are associated with the formation of scar tissues (12). Notably, miR-145 was revealed to be closely associated with tumorigenesis, myocardial injury and angiogenesis (13). For example, Yuan et al (14) demonstrated that miR-145 regulates myocardial ischemic injury by inhibiting the expression of cluster of differentiation (CD)40. Additionally, reduced miR-145 expression in peripheral blood has clinical diagnostic value in patients with coronary heart disease (15). miR-145 inhibits the proliferation and migration of lung cancer cells by regulating the expression of cadherin-2 (16). The aforementioned study also demonstrated that miR-145 is associated with the occurrence and development of hypertrophic scars (16). Zhu et al (17) reported that a peroxisome proliferator-activated receptor-agonist upregulates the expression of miR-145, and inhibits the TGF- $\beta$ /SMAD3 signaling pathway and hypertrophic scar formation. However, the target genes of miR-145 associated with the regulation of hypertrophic scars and the expression of miR-145 in hypertrophic scar tissues remain unclear. In the present study, the expression of miR-145 in hypertrophic scar tissues was measured and its mechanism of action was investigated.

\section{Materials and methods}

Patients. A total of 36 patients (21 males and 15 females) who were diagnosed with hypertrophic scars and received dermoplasty between May 2013 and June 2016 at The First Affiliated Hospital (Hangzhou, China) were included in the present study. The age range of the patients was 23-43 years and hyperplasia duration was between 3 months and 2 years. Skin damage sites exhibited hyperemia redness and lesions that did not exceed wound surface. The inclusion criteria were: i) Patients with hypertrophic scar tissues identified by clinicians; ii) patients without pituitary or adrenal diseases, infectious diseases, skin diseases, immune diseases, local infection or ulcer; and iii) patients not currently taking prescribed treatments. Patients with other tumors, chronic basic disease or a long history of drug intake were excluded. Hypertrophic scar tissues collected from all patients were used as the experimental group. For the control group, normal skin tissues from the hypogastrium of respective patients were used. The tissues were frozen and stored in liquid nitrogen. All procedures were approved by the Ethics Committee of Zhejiang University (Hangzhou, China). Written informed consent was obtained from all patients or their families.

Cells. Collected hypertrophic scar and control tissue samples were washed with PBS containing $100 \mu / \mathrm{ml}$ penicillin and streptomycin 3 times prior to removing epidermal and adipose tissues. The samples were cut into $1 \mathrm{~mm}^{3}$ sections, and washed with PBS containing $100 \mu / \mathrm{ml}$ penicillin and streptomycin 2 times. Subsequently, the samples were placed into culture flasks, and Dulbecco's modified Eagle medium (DMEM; Thermo Fisher Scientific, Inc., Waltham, MA, USA) was added prior to incubation at $37^{\circ} \mathrm{C}$ and $5 \% \mathrm{CO}_{2}$. After $2 \mathrm{~h}$, $2 \mathrm{ml}$ DMEM containing 10\% fetal bovine serum (Thermo Fisher Scientific, Inc.) was added into the flasks. One week later, spindle shape fibroblasts were observed around the tissue samples in the flasks under a light microscope (magnification, $\mathrm{x} 200$ ). Next, the tissues and culture medium were discarded, and fresh DMEM with $10 \%$ fetal bovine serum was added to the remaining fibroblasts in the culture flask. Once the cells reached $80 \%$ confluence, they were passaged and cells in 3-5 passages were used for subsequent experiments. A strain of hypertrophic scar fibroblasts (HSFB) and a strain of normal colonic fibroblasts (NCFB) were obtained.

Prior to transfection, fibroblasts in log-phase growth $\left(2 \times 10^{5}\right)$ were seeded onto 24 -well plates containing antibiotic-free DMEM supplemented with $10 \%$ fetal bovine serum and cultured at $37^{\circ} \mathrm{C}$. Once the cells reached $70 \%$ confluence, the cells were collected for transfection.

Transfection. In the first vial, $1.5 \mu 1 \mathrm{miR}$-negative control (NC group; $20 \mathrm{pmol} / \mu \mathrm{l}$; Hanbio Biotechnology Co., Ltd., Shanghai, China), miR-145 inhibitors (20 pmol $/ \mu 1$; the inhibitor group; 5'-GGAUUCCTGGAAATACTGTTCT-3'; Hanbio Biotechnology Co., Ltd.) or mimics ( $20 \mathrm{pmol} / \mu 1$; the mimics group; 5'-GTCCAGTTTTCCCAGGAATCCCT-3'; Hanbio Biotechnology Co., Ltd.) was mixed with $50 \mu$ 1 Opti-MEM medium (Thermo Fisher Scientific, Inc.). In another vial, $1 \mu$ l Lipofectamine ${ }^{\circledR} 3000$ Transfection Reagent (Thermo Fisher Scientific, Inc.) was mixed with $50 \mu 1$ Opti-MEM medium. The aforementioned two vials were incubated at room temperature for $5 \mathrm{~min}$, and then combined prior to incubation for $20 \mathrm{~min}$ at room temperature. The mixtures were subsequently added to the cells in respective groups at room temperature. A total of $6 \mathrm{~h}$ later, the medium was replaced with DMEM containing $10 \%$ fetal bovine serum. After culturing at $37^{\circ} \mathrm{C}$ for $48 \mathrm{~h}$, the cells were collected for further assays.

For rescue experiments, fibroblasts $\left(1 \times 10^{5}\right.$ cells $)$ in the miR-145 mimics and inhibitors groups were seeded onto 24-well plates containing antibiotic-free DMEM supplemented with $10 \%$ fetal bovine serum at $37^{\circ} \mathrm{C}$. Once the cells reached $60 \%$ confluence, fibroblasts in the miR-145 mimics and inhibitors groups were transfected with $0.5 \mu \mathrm{l}$ pLKO.1-sh-sox 9 and pcDNA3.1-SOX-9 plasmids (Hanbio Biotechnology Co., Ltd.), respectively, using Lipofectamine ${ }^{\circledR} 3000$, and named the miR-145 mimics + pLKO.1-sh-sox9 group and the miR-145 inhibitor + pcDNA3.1-SOX-9 group. After incubation at $37^{\circ} \mathrm{C}$ and under $5 \% \mathrm{CO}_{2}$ for $6 \mathrm{~h}$, the medium was changed to fresh DMEM containing $10 \%$ fetal bovine serum prior to continued cultivation for $72 \mathrm{~h}$.

Reverse transcription-quantitative polymerase chain reaction (RT-qPCR) analysis. Hypertrophic scar and control tissues $(100 \mathrm{mg})$ were ground into powder using liquid nitrogen and lysed with $1 \mathrm{ml} \mathrm{TRIzol}{ }^{\mathrm{TM}}$ Reagent (Thermo Fisher Scientific, Inc.). Then, total RNA was extracted using the phenol chloroform method (18). The purity of RNA was determined by the A260/A280 ratio using ultraviolet spectrophotometry (NanoDrop $^{\text {TM }}$ 2000; Thermo Fisher Scientific, Inc.). cDNA was obtained by reverse transcription from $1 \mu \mathrm{D}$ RNA and stored at $-20^{\circ} \mathrm{C}$. Reverse transcription was performed using miScript II RT kit (Qiagen GmbH, Hilden, Germany) following the manufacturer's protocol.

The expression of miRNA was determined by miScript $\mathrm{SYBR}^{\circledR}$ Green PCR kit (Qiagen $\mathrm{GmbH}$ ) using U6 (forward, 5'-CTCGCTTCGGCAGCACA-3'; reverse, 5'-AACGCTTCA 
CGAATTTGCGT-3') as an internal reference. The reaction mixture $(20 \mu 20$ contained $10 \mu$ l RT-qPCR-Mix, $0.5 \mu \mathrm{M}$ upstream primer (miR-145, 5'-GTCCAGTTCCCAGGAATC CCT-3'), $0.5 \mu$, downstream universal primer (provided in PCR kit), $2 \mu \mathrm{k}$ cDNA and $7 \mu \mathrm{c}$ double-distilled $\mathrm{H}_{2} \mathrm{O}$, and was performed using an iQ5 thermocycler (Bio-Rad Laboratories, Inc., Hercules, CA, USA). The reaction protocol was as follows: Initial denaturation at $95^{\circ} \mathrm{C}$ for $10 \mathrm{~min}$, and 40 cycles of $95^{\circ} \mathrm{C}$ for $1 \mathrm{~min}$ and $60^{\circ} \mathrm{C}$ for $30 \mathrm{sec}$. The $2^{-\Delta \Delta \mathrm{Cq}}$ method (19) was used to calculate the relative expression of miR-145 by comparing it against the expression of the internal reference. Each sample was tested in triplicate.

Cell counting Kit (CCK)-8 assay. Cells in each group were seeded at a density of 2,000 cells/well in 96 -well plates at $37^{\circ} \mathrm{C}$. At 0, 24, 48 and 72 h, $20 \mu \mathrm{l} \mathrm{CCK-8} \mathrm{(5} \mathrm{g/l;} \mathrm{Beyotime} \mathrm{Institute}$ of Biotechnology, Shanghai, China) was added to the cells and incubated at $37^{\circ} \mathrm{C}$ for $2 \mathrm{~h}$. The absorbance of each well was measured at $490 \mathrm{~nm}$ and cell proliferation curves were plotted. Each group was tested in 3 replicate wells and the mean value was calculated.

Flow cytometry. Cells in each group were seeded onto 12-well plates in triplicate at a density of $2 \times 10^{5}$ cells/well at $37^{\circ} \mathrm{C}$. A total of $24 \mathrm{~h}$ later, the cells were harvested and the cell cycle was determined using Cycletest ${ }^{\mathrm{TM}}$ Plus DNA Reagent kit (BD Biosciences, Franklin Lakes, NJ, USA) according to the manufacturer's protocol. Briefly, the cells were incubated with $150 \mu \mathrm{i}$ liquid $\mathrm{A}$ at room temperature for $10 \mathrm{~min}$, and $150 \mu \mathrm{l}$ liquid $\mathrm{B}$ for a further $10 \mathrm{~min}$. Cells were subsequently incubated with $120 \mu 1$ liquid $\mathrm{C}$ in the dark for $10 \mathrm{~min}$ before analysis with a FACSCanto II Flow Cytometer (BD Biosciences). The result was analyzed using ModFit software (version 3.2; Verity Software House, Inc., Topsham, ME, USA).

To detect cell apoptosis, FITC Annexin V Apoptosis Detection Kit I (BD Biosciences) was used. Briefly, the cells were trypsinized and centrifuged at $500 \mathrm{xg}$ at room temperature for $3 \mathrm{~min}$. Then, the cells were washed with precooled PBS twice and centrifuged at $500 \mathrm{xg}$ at room temperature for $3 \mathrm{~min}$. Following the removal of the supernatant, $5 \mu \mathrm{l}$ Annexin $\mathrm{V}$-fluorescein isothiocyanate and $5 \mu \mathrm{n}$ propidine iodide staining solutions were added to the cells, and the samples were incubated in dark at room temperature for $15 \mathrm{~min}$ prior to flow cytometry. The result was analyzed using ModFit software.

Matrigel assay. Matrigel was thawed at $4^{\circ} \mathrm{C}$ overnight and diluted with serum-free DMEM medium (dilution 1:2). The mixture (50 $\mu \mathrm{o}$ was evenly smeared into the upper chamber of Transwell plates (Merck KGaA, Darmstadt, Germany) and incubated at $37^{\circ} \mathrm{C}$ for $1 \mathrm{~h}$. Following the solidification of the gel, $1 \times 10^{5}$ cells from each group were seeded into the upper chamber containing $200 \mu \mathrm{l}$ serum-free DMEM medium. In addition, $500 \mu 1$ DMEM medium supplemented with $10 \%$ fetal bovine serum was added into the lower chamber. After $24 \mathrm{~h}$, the chamber was removed and the cells in the upper chamber were discarded. Following fixation at room temperature with $4 \%$ formaldehyde for $10 \mathrm{~min}$, the membrane was stained using the Giemsa method (20) for light microscopic observation of 5 random fields (magnification, 200x). The number of Transwell cells was calculated for the evaluation of cell invasion ability. All procedures were carried out on ice with pipetting tips being precooled at $4^{\circ} \mathrm{C}$.

Western blotting. Cells in each group were trypsinized and collected. Then, precooled Radio-Immunoprecipitation Assay (RIPA) lysis buffer (600 $\mu \mathrm{l} ; 50 \mathrm{mM}$ Tris-base, $1 \mathrm{mM}$ EDTA, $150 \mathrm{mM} \mathrm{NaCl}, 0.1 \%$ sodium dodecyl sulfate, $1 \%$ TritonX-100, $1 \%$ sodium deoxycholate; Beyotime Institute of Biotechnology) was added to the samples. Following lysis for $30 \mathrm{~min}$ on ice, the mixture was centrifuged at $12,000 \mathrm{xg}$ and $4^{\circ} \mathrm{C}$ for $10 \mathrm{~min}$. The supernatant was used to determine protein concentration by bicinchoninic acid protein concentration determination kit (cat. no. RTP7102; Real-Times Biotechnology Co., Ltd., Beijing, China). Protein samples (6 $\mu 1 /$ lane) were then mixed with $5 \mathrm{X}$ SDS loading buffer prior to denaturation in a boiling water bath for $10 \mathrm{~min}$. The samples were then separated by $10 \%$ SDS-PAGE (100V). The resolved proteins were transferred to polyvinylidene difluoride membranes on ice $(250 \mathrm{~mA}, 1 \mathrm{~h})$ and blocked with $5 \%$ skimmed milk at room temperature for $1 \mathrm{~h}$. Then, the membranes were incubated with rabbit anti-human SOX-9 polyclonal (1:1,000; cat. no. sc-20095) and GAPDH (1:4,000; cat. no. sc-293335; both Santa Cruz Biotechnology, Inc., Dallas, TX, USA) primary antibodies at $4^{\circ} \mathrm{C}$ overnight. Following extensive washing with Tween 20 in PBS 5 times (5 min each), the membranes were incubated with goat anti-rabbit (cat. no. sc-2007) and goat anti-mouse (cat. no. sc-2039) horseradish peroxidase-conjugated secondary antibodies (1:4,000; Santa Cruz Biotechnology, Inc.) for $1 \mathrm{~h}$ at room temperature prior to washing with Tween 20 in PBS 5 times (5 min each). Subsequently, the membrane was developed with the enhanced chemiluminescence detection kit (Sigma-Aldrich; Merck KGaA) for imaging. Image lab software (version 3.0; Bio-Rad Laboratories, Inc.) was used to analyze imaging signals. The relative content of SOX-9 protein was expressed as a SOX-9/GAPDH ratio.

Dual luciferase reporter assay. According to bioinformatics results (www.targetscan.com), wild-type (WT) and mutant seed regions of miR-145 in the 3'-UTR of SOX-9 gene were chemically synthesized in vitro. The seed regions were cut with Spe-1 and HindIII restriction sites, and then cloned into pMIR-REPORT luciferase reporter plasmids (Thermo Fisher Scientific, Inc.). Plasmids $(0.5 \mu \mathrm{g})$ with WT or mutant 3'-UTR DNA sequences were co-transfected with miR-145 mimics into 293T cells (Type Culture Collection of the Chinese Academy of Sciences, Shanghai, China) using Lipofectamine 3000 (Thermo Fisher Scientific, Inc.). After cultivating at $37^{\circ} \mathrm{C}$ for $24 \mathrm{~h}$, the cells were lysed using dual luciferase reporter assay kit (Promega Corporation, Madison, WI, USA) according to the manufacturer's protocol, and fluorescence intensity was measured using a GloMax 20/20 luminometer (Promega Corporation). Using Renilla luciferase activity as an internal reference, the luciferase activity of each group of cells was measured.

Statistical analysis. Results were analyzed using SPSS 17.0 statistical software (SPSS, Inc., Chicago, IL, USA). Data are presented as mean \pm standard deviation. The differences between datasets containing multiple groups were analyzed 
using one-way analysis of variance followed by Tukey's test. Differences between datasets containing two groups were analyzed using a Student's t-test. $\mathrm{P}<0.05$ was considered to indicate a statistically significant difference.

\section{Results}

Expression of miR-145 is reduced in hypertrophic tissues and fibroblasts. To measure the expression of miR-145 in hypertrophic scar tissues and fibroblasts, RT-qPCR analyses were performed. The data demonstrated that the expression of miR-145 in hypertrophic scar tissues was significantly reduced compared with that of the control tissues ( $\mathrm{P}<0.05$; Fig. 1A). Similarly, the expression of miR-145 in HSFB was significantly lower than that in NCFB $(\mathrm{P}<0.05$; Fig. 1B). These results demonstrate that expression of miR-145 is reduced in hypertrophic tissues and fibroblasts.

Overexpression of miR-145 inhibits the proliferation of fibroblasts in vitro. To determine fibroblast proliferation, a CCK-8 assay was performed. The data revealed that the absorbance of HSFB transfected with miR-145 mimics was significantly lower than that of HSFB transfected with miR-NC at 24, 48 and $72 \mathrm{~h}$ (all $\mathrm{P}<0.05$; Fig. 2A). In addition, the absorbance of NCFB transfected with miR-145 inhibitor was significantly higher than that of NCFB transfected with miR-NC at 24, 48 and $72 \mathrm{~h}$ (all $\mathrm{P}<0.05$; Fig. 2B). These results indicate that miR-145 overexpression inhibits the proliferation of fibroblasts in vitro.

Overexpression of miR-145 delays G1/S phase transition of fibroblasts. For cell cycle determination, flow cytometry was performed. The data revealed that G1/S phase transition of HSFB transfected with miR-145 mimics was reduced $(\mathrm{P}<0.05$; Fig. 3A), whereas G1/S phase transition of NCFB transfected with miR-145 inhibitors was promoted ( $\mathrm{P}<0.05$; Fig. 3B). These result suggest that overexpression of miR-145 delays G1/S phase transition of fibroblasts.

Overexpression of miR-145 promotes apoptosis of fibroblasts. To examine cell apoptosis, flow cytometry was performed. The data demonstrated that the apoptotic rate of HSFB transfected with miR-145 mimics was significantly higher than that in the miR-NC group ( $\mathrm{P}<0.05$; Fig. 4A), whereas the apoptotic rate of NCFB transfected with miR-145 inhibitors was significantly lower than that in the miR-NC group $(\mathrm{P}<0.05$; Fig. 4B). These results indicate that overexpression of miR-145 promotes the apoptosis of fibroblasts.

Overexpression of miR-145 suppresses invasion of fibroblasts. To determine the invasion ability of fibroblasts, a Matrigel assay was employed. The data revealed that the number of HSFB that crossed the membrane in the miR-145 mimics group was significantly lower than that in the miR-NC group $(\mathrm{P}<0.05$; Fig. 5A). In addition, the number of $\mathrm{NCFB}$ that crossed the membrane in the miR-145 inhibitor group was significantly higher than that in the miR-NC group $(\mathrm{P}<0.05$; Fig. 5B). These results suggest that overexpression of miR-145 suppresses invasion of fibroblasts.

Overexpression of miR-145 may inhibit SOX-9 protein expression. To measure the expression of SOX-9 protein, western
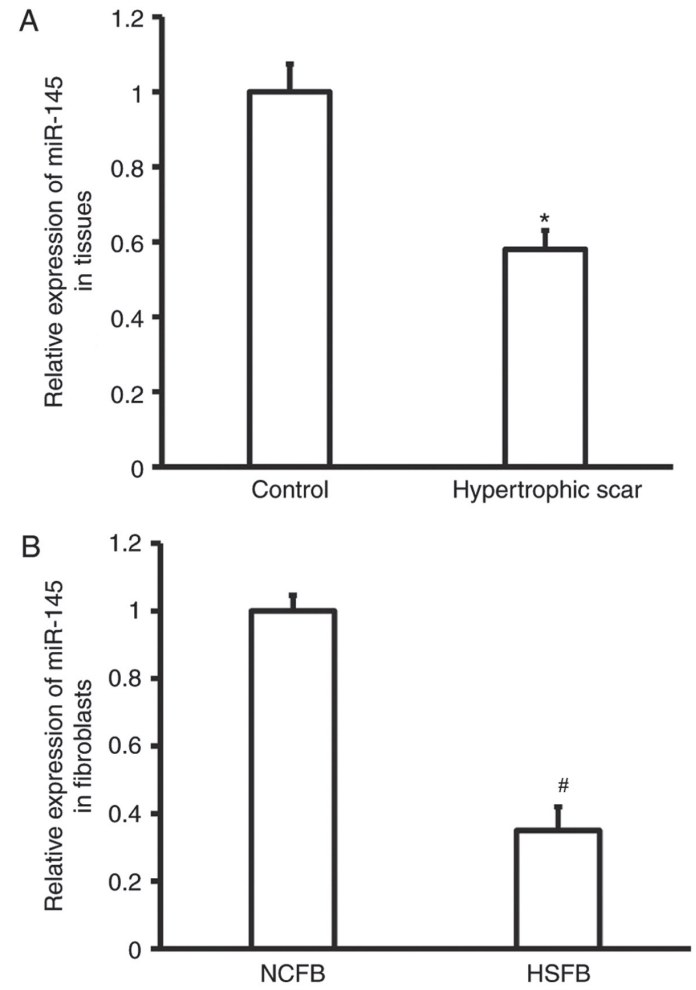

Figure 1. miR-145 is decreased in hypertrophic scar tissues and fibroblasts. The expression of miR-145 in (A) hypertrophic scar tissues and (B) fibroblasts were measured via reverse transcription-quantitative polymerase chain reaction. " $\mathrm{P}<0.05$ vs. control; ${ }^{\#} \mathrm{P}<0.05$ vs. NCFB. miR, microRNA; NCFB, normal colonic fibroblasts; HSFB, hypertrophic scar fibroblasts.

A
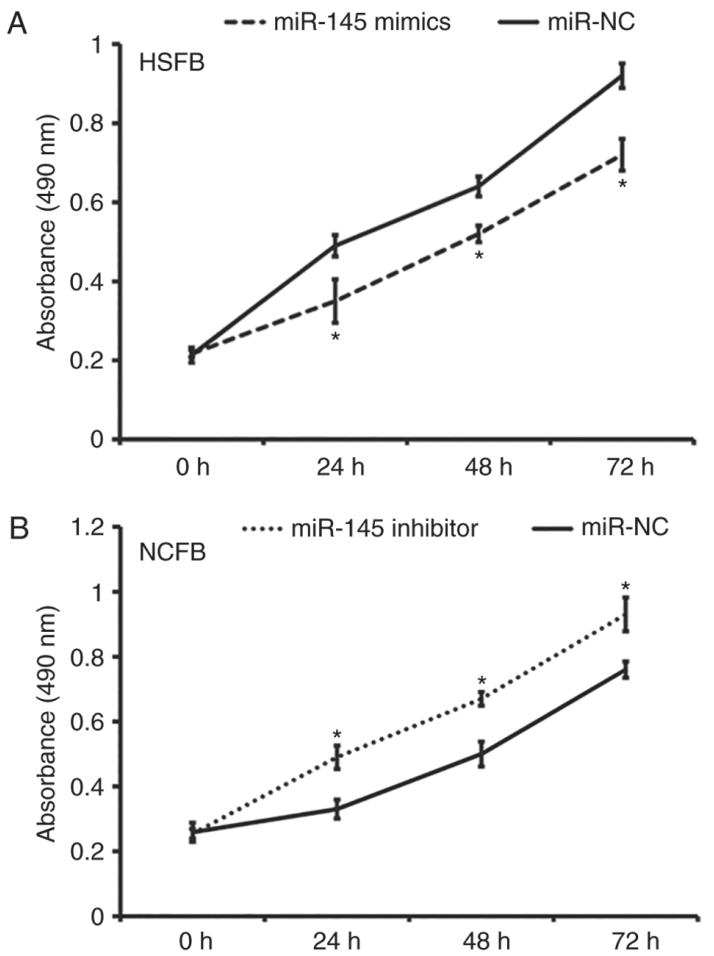

Figure 2. miR-145 increases the proliferation of fibroblasts. A Cell Counting Kit-8 assay was performed to determine the proliferation of cells. (A) Absorbance of HSFB transfected with miR-NC or miR-145 mimics. (B) Absorbance of NCFB transfected with miR-NC or miR-145 inhibitors ${ }^{*} \mathrm{P}<0.05$ vs. miR-NC group at the same time point. miR, microRNA; NC, negative control; HSFB, hypertrophic scar fibroblasts; NCFB, normal colonic fibroblasts. 

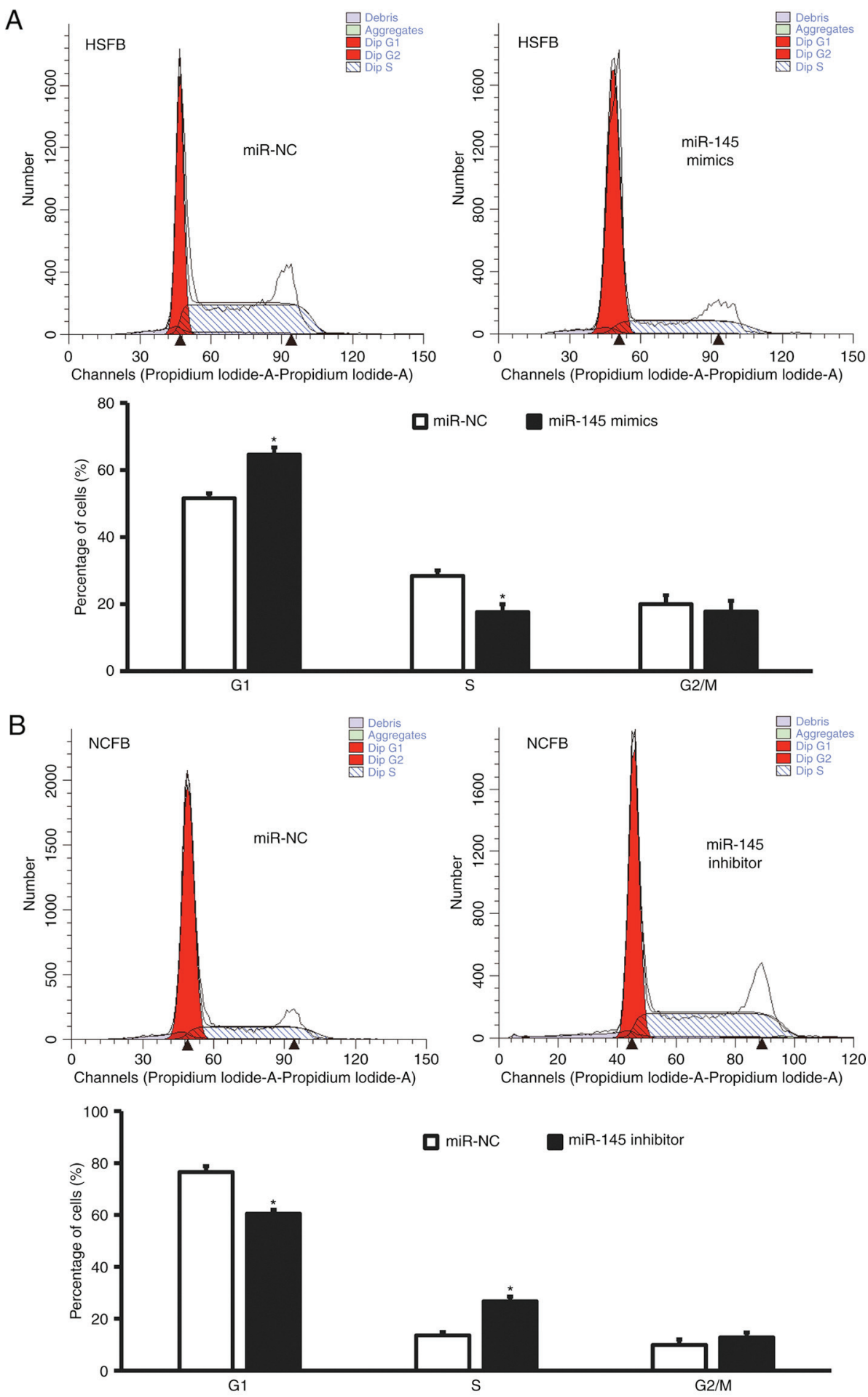

Figure 3. miR-145 slows the G1/S phase transition of fibroblasts. Flow cytometry was performed to determine cell cycles. (A) Percentage of HSFB transfected with miR-145 mimics or miR-NC in each cell phase. (B) Percentage of NCFB transfected with miR-145 inhibitors or miR-NC in each cell phase. ${ }^{*} \mathrm{P}<0.05$ vs. miR-NC group in same phase. miR, microRNA; NC, negative control; HSFB, hypertrophic scar fibroblasts; NCFB, normal colonic fibroblasts.

blotting was performed. The data demonstrated that the expression of SOX-9 in hypertrophic fibroblasts transfected with miR-145 mimics was significantly reduced compared with that in hypertrophic fibroblasts transfected with miR-NC $(\mathrm{P}<0.05$; Fig. 6A). By contrast, the expression of SOX-9 in normal fibroblasts transfected with miR-145 inhibitors was significantly enhanced compared with that in normal fibroblasts transfected with miR-NC ( $\mathrm{P}<0.05$; Fig. 6B). The results indicate that overexpression of miR-145 inhibits SOX-9 protein expression.

Expression of SOX-9 can reverse the effects of miR-145 on the proliferation, cell cycle, apoptosis and invasion of fibroblasts. To test whether miR-145 affects the biological functions of fibroblasts by regulating the expression of SOX-9 protein, CCK-8, flow cytometry and Matrigel assay were performed. 

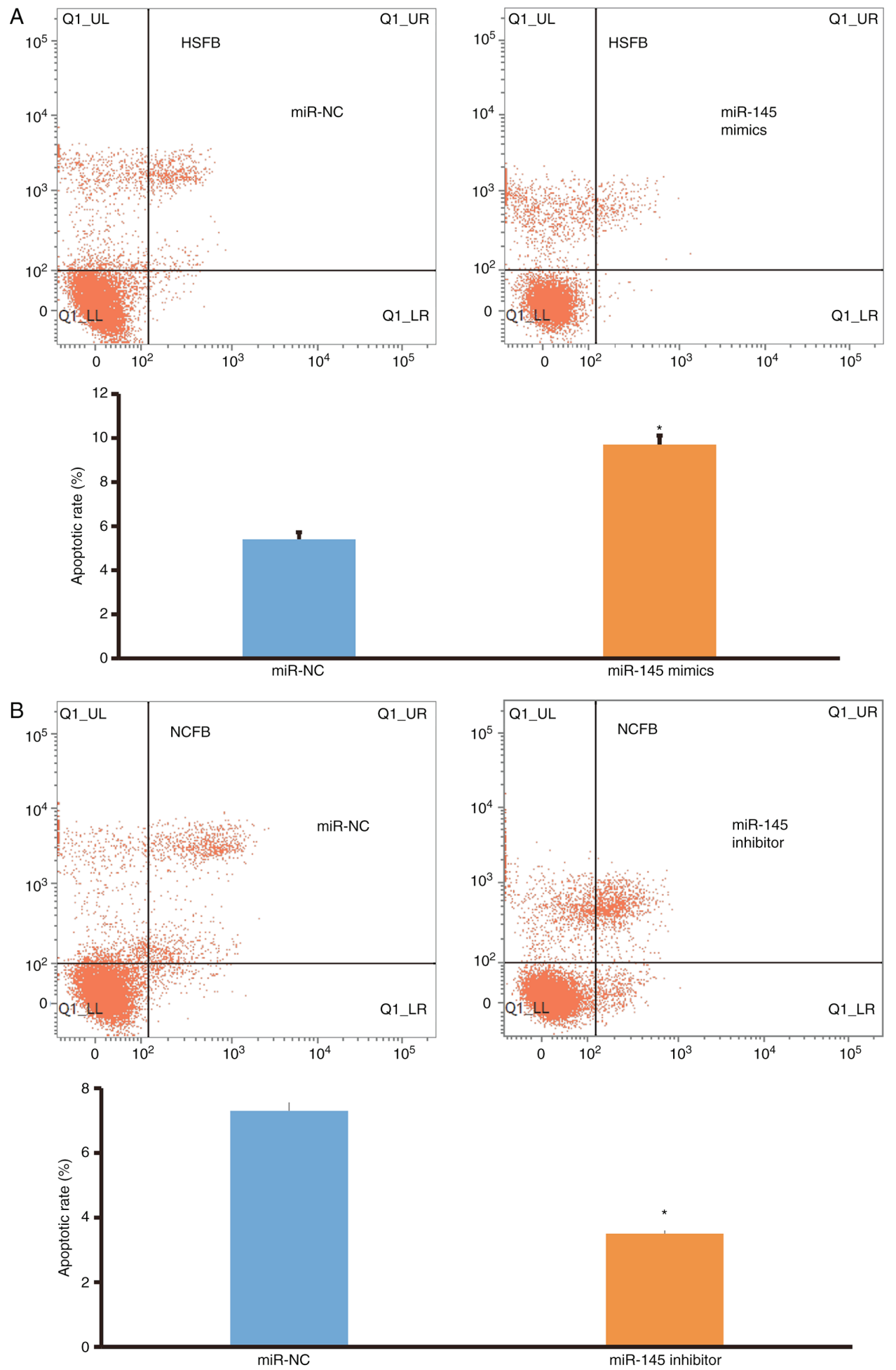

Figure 4. miR-145 increases the apoptosis of fibroblasts. Flow cytometry was used to determine apoptosis. (A) The apoptotic rate of HSFB transfected with miR-145 mimics or miR-NC. (B) The apoptotic rate of NCFB transfected with miR-145 inhibitors or miR-NC. "P<0.05 vs. miR-NC group. miR, microRNA; $\mathrm{NC}$, negative control; HSFB, hypertrophic scar fibroblasts; NCFB, normal colonic fibroblasts.

These assays analyzed the proliferation, cell cycle determination, apoptosis rate and invasion of fibroblasts transfected with miR-145 mimics or inhibitors following the overexpression of the SOX-9 protein by a plasmid containing the SOX-9 gene or the silencing of the SOX-9 protein by a plasmid containing a SOX-9 shRNA, respectively. The CCK- 8 assay revealed that the downregulation of SOX-9 expression by the SOX-9 shRNA in miR-145 inhibitor-transfected NCFB significantly decreased cell proliferation compared with NCFB transfected with miR-145 inhibitors alone and miR-NC $(\mathrm{P}<0.05$; Fig. 7A), whereas the overexpression of SOX-9 by the plasmid containing the SOX-9 gene in miR-145 mimic-transfected HSFB significantly decreased cell proliferation compared with HSFB transfected with miR-145 mimics alone and miR-NC (P<0.05; Fig. 7B). Flow cytometry demonstrated that the overexpression of SOX-9 significantly enhanced the G1/S 
A
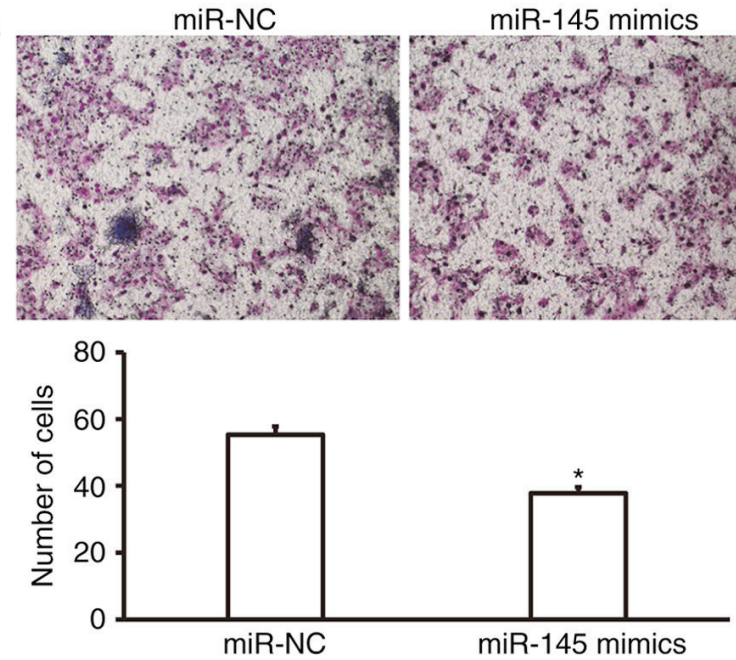

B
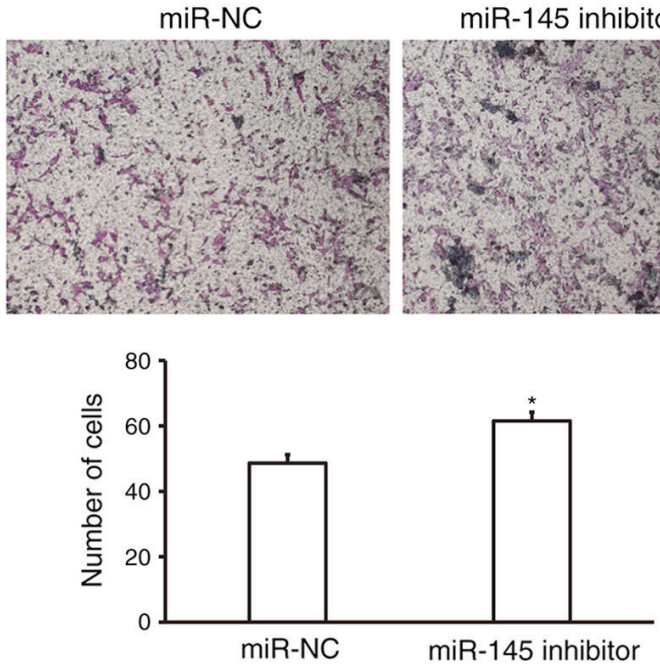

Figure 5. Effect of miR-145 on the invasion of fibroblasts. A Matrigel assay was used to determine the invasion ability of fibroblasts. (A) Representative images and quantitative analysis of hypertrophic scar fibroblasts transfected with miR-145 mimics or miR-NC that invaded the lower chamber (magnification, $\mathrm{x} 100$ ). (B) Representative images and quantitative analysis of migrated normal colonic fibroblasts transfected with miR-145 inhibitors or miR-NC that invaded the lower chamber. "P<0.05 vs. miR-NC group. miR, microRNA; NC, negative control.

A
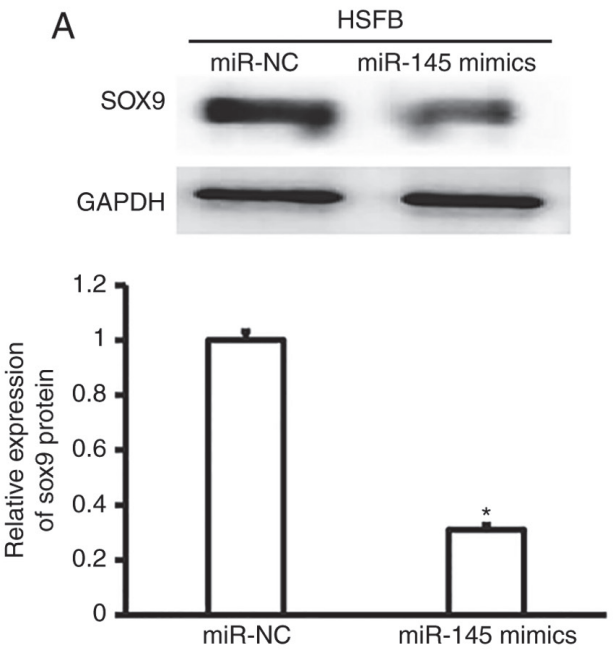

B
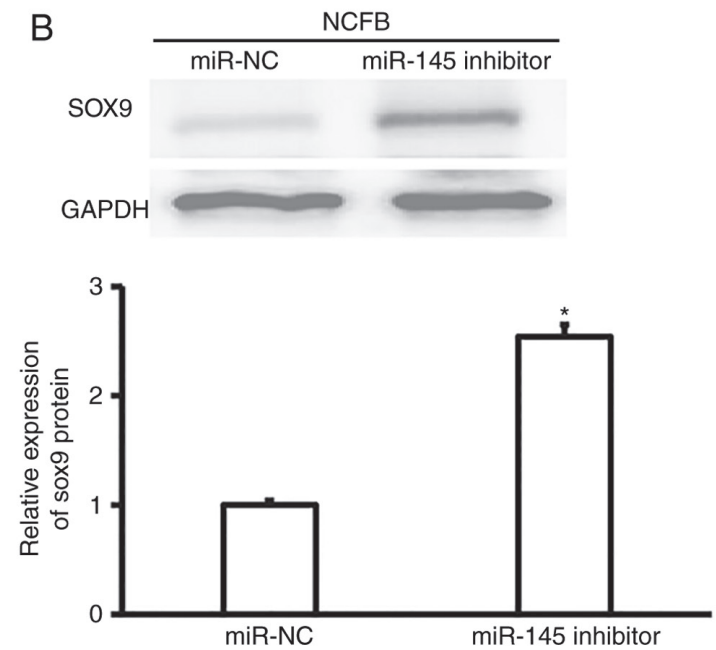

Figure 6. miR-145 decreases the expression of the SOX-9 protein in fibroblasts. The expression of SOX-9 protein was determined by western blotting. (A) The expression of SOX-9 protein in HSFB transfected with miR-145 mimics or miR-NC. (B) The expression of SOX-9 protein in NCFB transfected with miR-145 inhibitors or miR-NC. "P<0.05 vs. miR-NC group. miR, microRNA; NC, negative control; HSFB, hypertrophic scar fibroblasts; NCFB, normal colonic fibroblasts; SOX-9, transcription factor SOX-9.

phase transition of HSFB transfected with miR-145 mimics compared with HSFB transfected with miR-145 mimics alone and miR-NC $(\mathrm{P}<0.05$; Fig. 7C), whereas downregulation of SOX-9 expression significantly decreased the G1/S phase transition of NCFB transfected with miR-145 inhibitors compared with NCFB transfected with miR-145 inhibitors alone and miR-NC ( $\mathrm{P}<0.05$; Fig. 7D). Overexpression of SOX-9 significantly decreased the apoptotic rate of HSFB transfected with miR-145 mimics compared with HSFB transfected with miR-145 mimics alone and miR-NC $(\mathrm{P}<0.05$; Fig. 7E) and the downregulation of SOX-9 expression significantly decreased the apoptotic rate of NCFB transfected with miR-145 inhibitors compared with NCFB transfected with miR-145 inhibitors alone and miR-NC $(\mathrm{P}<0.05$; Fig. $7 \mathrm{~F})$. The Matrigel assay demonstrated that the overexpression of SOX-9 significantly increased the number of transmembrane HSFB transfected with miR-145 mimics compared with HSFB transfected with miR-145 mimics alone and miR-NC $(\mathrm{P}<0.05$; Fig. 7G) and the down-regulation of SOX-9 expression significantly decreased the number of transmembrane NCFB transfected with miR-145 inhibitors compared with NCFB transfected with miR-145 inhibitors alone and miR-NC $(\mathrm{P}<0.05$; Fig. $7 \mathrm{H})$. These results demonstrate that the expression of SOX-9 reverses the effects of miR-145 on the proliferation, cell cycle determination, apoptosis and invasion of fibroblasts.

miR-145 binds with the 3'-UTR of the SOX-9 mRNA to regulate SOX-9 protein expression. To identify the interaction between the seed region of miR-145 and the 3'-UTR of SOX-9 mRNA, a dual luciferase reporter assay was performed. The fluorescence value of cells co-transfected with miR-145 mimics and pMIR-REPORT-WT luciferase reporter plasmids 

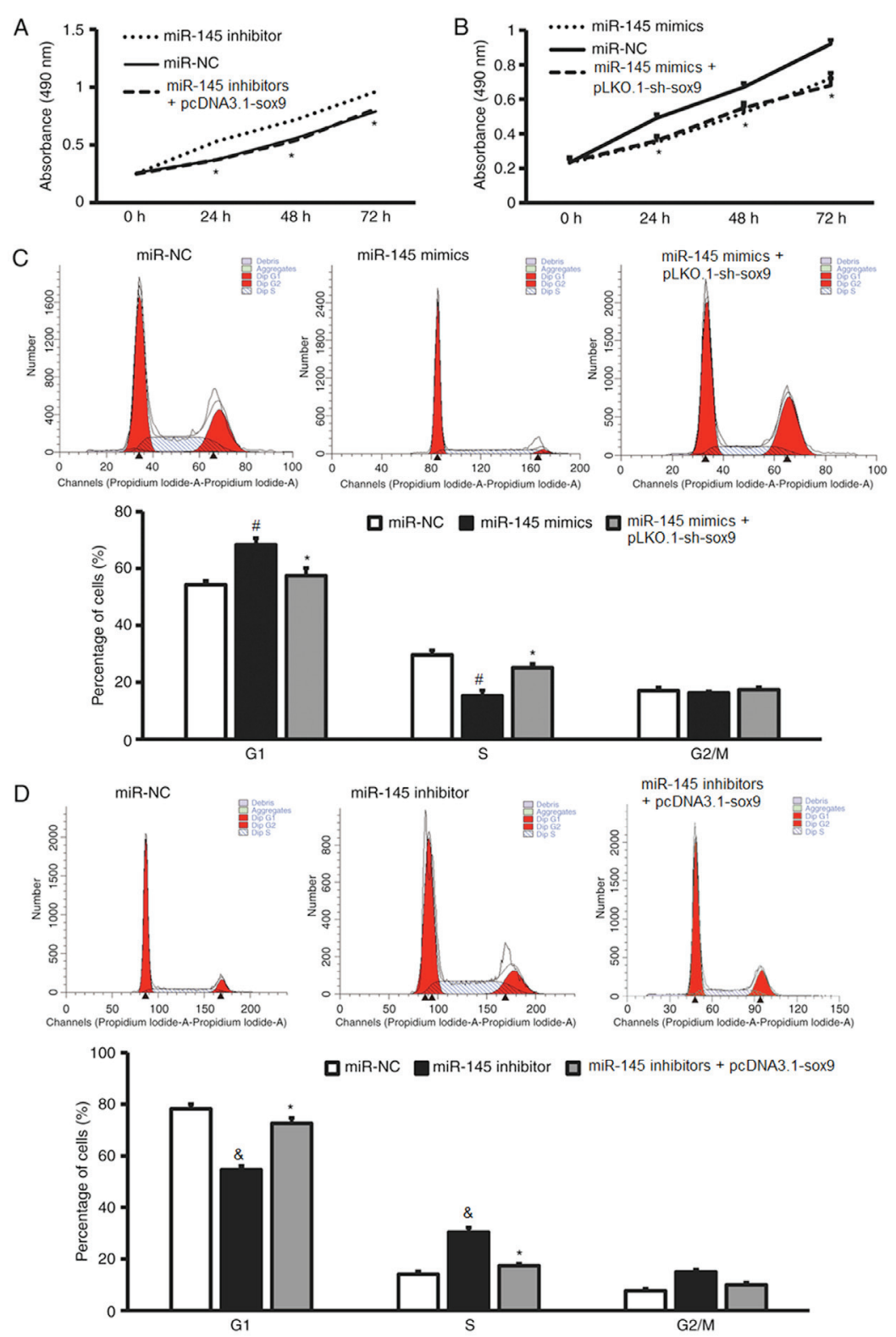

Figure 7. Increased SOX-9 expression reverses effect of miR-145 mimics on the proliferation, cell cycle, apoptosis and invasion of fibroblasts. (A) The proliferation of NCFB transfected with miR-145 inhibitors or miR-NC, as determined by a CCK-8 assay. (B) The proliferation of HSFB transfected with miR-145 mimics or miR-NC, as determined by a CCK-8 assay. (C) Flow cytomery results and the percentages of HSFB transfected with miR-NC, miR-145 mimics or miR-145 mimics + pLKO.1-sh-sox9 in each cell cycle phase. (D) Flow cytomery results and the percentages of NCFB transfected with miR-NC, miR-145 inhibitor or miR-145 inhibitors + pcDNA3.1-sox9 in each cell cycle phase. ${ }^{*} \mathrm{P}<0.05$ vs. miR-NC; ${ }^{\#} \mathrm{P}<0.05$ vs. miR-145 mimics + pLKO.1-sh-sox9 group; ${ }^{\circledR} \mathrm{P}<0.05$ vs. miR-145 inhibitors + pcDNA3.1-sox9 group.

was significantly lower than that in the negative control group $(\mathrm{P}<0.05$; Fig. 8). By contrast, no significant differences were identified between the fluorescence value of cells co-transfected with miR-145 mimics and pMIR-REPORT-mutant luciferase reporter plasmids, and that of the NC group. The result indicates that the miR-145 seed region can bind with the 3'-UTR of the SOX-9 mRNA to regulate SOX-9 protein expression.

\section{Discussion}

Fibroblasts are the major effector cells in wound healing (21). In the process of hypertrophic scar formation, fibroblasts typically exhibit excessive proliferation, enhanced migration and inhibited apoptosis, and secrete abundant extracellular matrix $(22,23)$. It was previously identified that miRNA molecules serve important roles in the biological functions of hypertrophic scar fibroblasts, but their mechanisms of action requires further elucidation (24). In the present study, it was identified that miR-145 expression is significantly downregulated in hypertrophic scar tissues, suggesting that miR-145 may be associated with the occurrence and development of hypertrophic scar. In vitro experiments revealed that miR-145 inhibits the proliferation, invasion and G1/S phase transition of fibroblasts, and promotes the apoptosis of fibroblasts. Bioinformatics and molecular biology experiments demonstrated that miR-145 exerts these effects by regulating SOX-9 gene expression. Therefore, miR-145 may be a potential therapeutic target in the treatment of hypertrophic scar. 

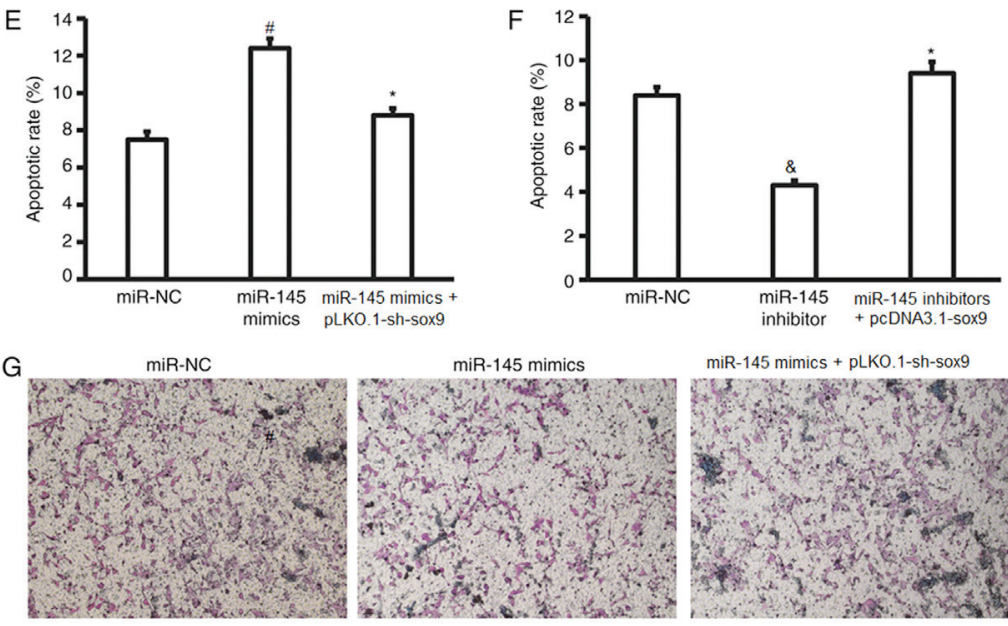

miR-145 mimics + pLKO.1-sh-sox9
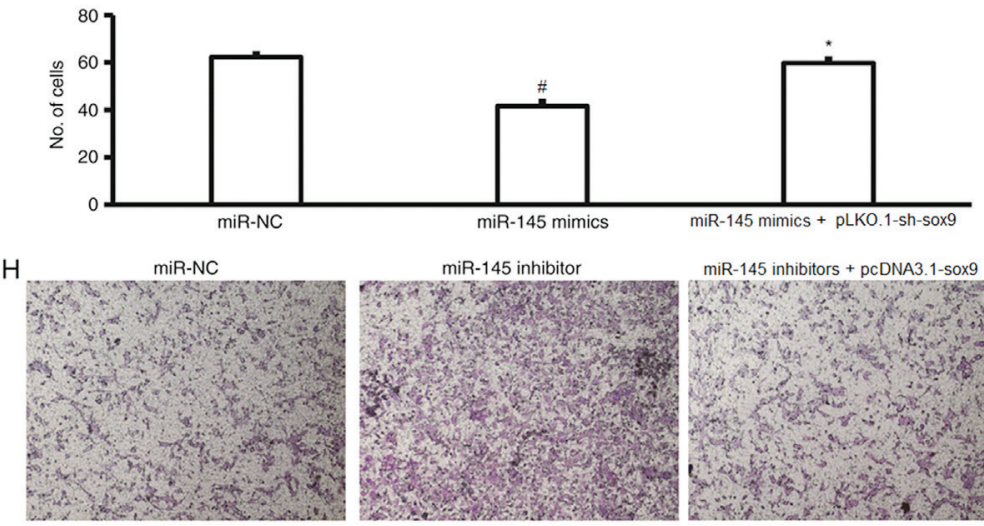

miR-145 inhibitor

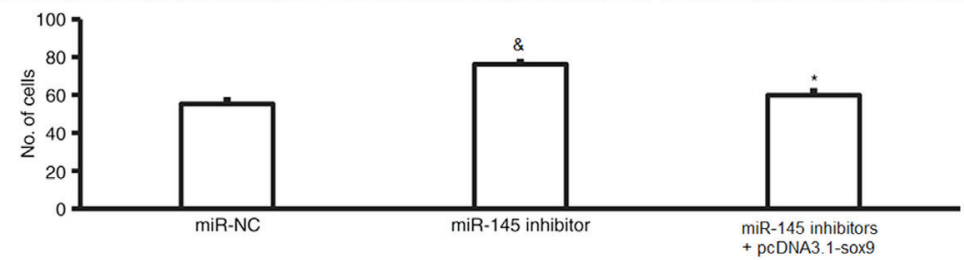

Figure 7. Continued. Increased SOX-9 expression reverses effect of miR-145 mimics on the proliferation, cell cycle, apoptosis and invasion of fibroblasts. (E) The apoptotic rate of HSFB transfected with miR-NC, miR-145 mimics or miR-145 mimics + pLKO.1-sh-sox9, as determined by flow cytometry. (F) The apoptotic rate of NCFB transfected with miR-NC, miR-145 inhibitor or miR-145 inhibitors + pcDNA3.1-sox9, as determined by flow cytometry. (G) Matrigel invasion assay images and the number of transmembrane HSFB transfected with miR-NC, miR-145 mimics or miR-145 mimics + pLKO.1-sh-sox9. (H) Matrigel invasion assay images and the number of transmembrane NCFB transfected with miR-NC, miR-145 inhibitor or miR-145 inhibitors + pcDNA3.1-sox9. " $\mathrm{P}<0.05$ vs. miR-NC; " $\mathrm{P}<0.05$ vs. miR-145 mimics + pLKO.1-sh-sox9 group; ${ }^{\circledR} \mathrm{P}<0.05$ vs. miR-145 inhibitors + pcDNA3.1-sox9 group. CCK-8, Cell Counting Kit-8; miR, microRNA; NC, negative control; HSFB, hypertrophic scar fibroblasts; NCFB, normal colonic fibroblasts.

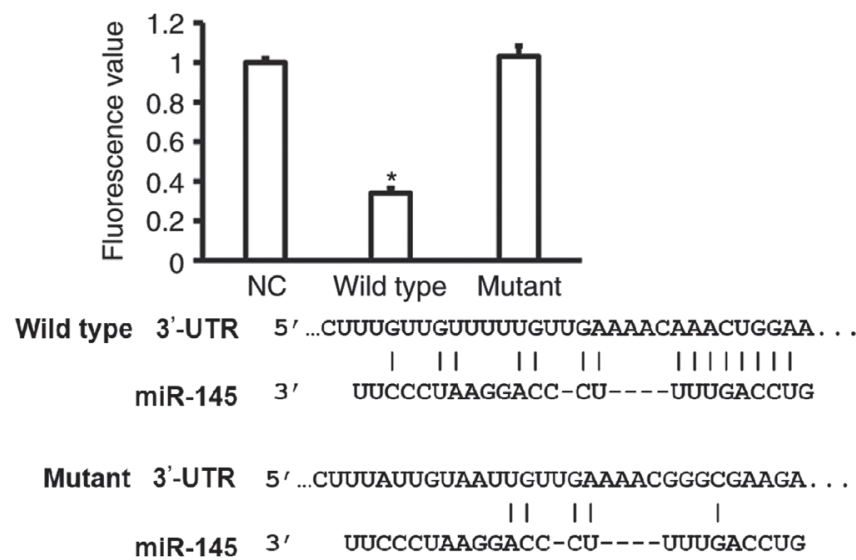

Figure 8. Identification of interaction between miR-145 and SOX-9 mRNA. A dual luciferase reporter assay was used to determine the interaction between miR-145 and SOX-9 mRNA. Plasmids with wild-type or mutant 3'-UTR DNA sequences were co-transfected with miR-145 mimics into $293 \mathrm{~T}$ cells. Following cultivation for $24 \mathrm{~h}$, cells were measured using a GloMax 20/20 luminometer. Using Renilla fluorescence activity as internal reference, the fluorescence values of each group of cells were measured. In addition, the seed regions of miR-145 and 3'-UTR of wild-type and mutant SOX-9 are presented. "P<0.05 vs. NC group. NC, negative control; miR, microRNA; UTR, untranslated region. 
As an important class of post-transcriptional regulators, miRNA molecules are widely associated with the proliferation, aging, apoptosis and migration of skin fibroblasts (25). For example, Xie et al (26) revealed that miR-377 facilitates the aging of skin fibroblasts by targeting the DNA (cytosine-5)-methyltransferase $1 \mathrm{mRNA}$. Zeng et al (27) demonstrated that miR-27b inhibits the activation of fibroblasts by regulating the TGF- $\beta$ signaling pathway. Li et al (28) reported that miR-19 inhibits the release of cytokines from fibroblasts by targeting Toll-like receptor 2 mRNA. In vitro experiments demonstrated that miR-145 expression in HSFB is significantly lower than that in NCFB, suggesting that miR-145 has regulatory roles in biological functions of fibroblasts. It was reported that miR-145 regulates the TGF- $\beta$ GF-rted that miR-145 regulates is significantly lower than that in NCFB, subcomponents by fibroblasts and the formation of hypertrophic scar (17). The aforementioned study is consistent with the results of the current study.

An miRNA may regulate multiple target genes (29). A recent study revealed that miR-145 targets SMAD3 expression and exerts its biological functions in fibroblasts (14). However, it remains unclear whether miR-145 regulates hypertrophic scar formation via other target mRNA. SOX-9 is a member of the SOX gene family, which is closely associated with the proliferation, apoptosis and differentiation of cells (30). For example, SOX-9 gene transcription regulates the activity of the wnt signaling pathway in intestinal epithelial stem cells (31). In addition, SOX-9 promotes the proliferation, migration and differentiation of multiple tumor cells, including lung cancer, thyroid carcinoma and gastric cancer (32). Furthermore, SOX-9 mRNA is regulated by several miRNA molecules. It was recently demonstrated that miR-105 inhibits the occurrence and development of glioma by targeting SOX-9 (33). miR-592 also suppresses the proliferation and metastasis of non-small cell lung cancer by downregulating the expression of SOX-9 (34). It was also reported that miR-124, miR-30a and miR-494 directly regulate the expression of the SOX-9 gene (35-37). In the present study, bioinformatics revealed that SOX-9 is a potential target gene of miR-145, and that miR-145 expression is negatively associated with SOX-9 expression. Notably, rescue experiments demonstrate that miR-145 exerts its biological functions in fibroblasts by regulating SOX-9. Importantly, dual luciferase reporter assay identified that SOX-9 is a direct target gene of miR-145.

In conclusion, the present study demonstrated that miR-145 expression is downregulated in hypertrophic scar tissues. In addition, miR-145 inhibited fibroblast proliferation and invasion, and promoted the apoptosis of fibroblasts by targeting SOX-9 expression. Therefore, it is suggested that miR-145 regulates the occurrence and development of hypertrophic scars. The results of the current study may provide a potential target for the clinical diagnosis and treatment of hypertrophic scars.

\section{Acknowledgements}

The authors would like to thank Dr Weifang Zhu from Department of Dermatology, The First Affiliated Hospital, Zhejiang University.

\section{Funding}

The present study was supported by Zhejiang University.

\section{Availability of data and materials}

The datasets used and/or analyzed during the current study are available from the corresponding author on reasonable request.

\section{Authors' contributions}

SW and JQ designed the current study. SW, CL and YY performed the experiments. SW, CL and JQ analyzed the data. SW and JQ interpreted results and prepared the manuscript. The final version of the manuscript has been read and approved by all authors.

\section{Ethics and consent to participate}

All procedures performed in the current study were approved by the Ethics Committee of Zhejiang University. Written informed consent was obtained from all patients or their families prior to enrollment.

\section{Patient consent for publication}

Written informed consent for the publication of associated data and accompanying images were obtained from all patients or their parents, guardians or next of kin.

\section{Competing interests}

The authors declare that they have no competing interests.

\section{References}

1. Füller $\mathbf{J}$ and Müller-Goymann CC: Anti-proliferative and anti-migratory effects of hyperforin in 2D and 3D artificial constructs of human dermal fibroblasts-A new option for hypertrophic scar treatment? Eur J Pharm Biopharm 126: 108-114, 2018.

2. Liu DQ, Li XJ and Wenj XJ: Effect of BTXA on inhibiting hypertrophic scar formation in a rabbit ear model. Aesthetic Plast Surg 41: 721-728, 2017.

3. Guo L, Xu K, Yan H, Feng H, Wang T, Chai L and Xu G: MicroRNA expression signature and the therapeutic effect of the microRNA21 antagomir in hypertrophic scarring. Mol Med Rep 15: 1211-1221, 2017.

4. Ai JW, Liu JT, Pei SD, Liu Y, Li DS, Lin HM and Pei B: The effectiveness of pressure therapy $(15-25 \mathrm{mmHg}$ ) for hypertrophic burn scars: A systematic review and meta-analysis. Sci Rep 7: 40185, 2017.

5. Chen YY, Lu YH, Ma CH, Tao WW, Zhu JJ and Zhang X: A novel elastic liposome for skin delivery of papain and its application on hypertrophic scar. Biomed Pharmacother 87: 82-91, 2017.

6. Carney BC, Liu Z, Alkhalil A, Travis TE, Ramella-Roman J, Moffatt LT and Shupp JW: Elastin is differentially regulated by pressure therapy in a porcine model of hypertrophic scar. J Burn Care Res 38: 28-35, 2017.

7. Li H, Yang L, Zhang Y and Gao Z: Kaempferol inhibits fibroblast collagen synthesis, proliferation and activation in hypertrophic scar via targeting TGF- $\beta$ receptor type I. Biomed Pharmacother 83: 967-974, 2016.

8. Dong JY, Song F, Liu YK and Wang XQ: Effects of severe hypoxia and low concentration of serum protein on the function of human hypertrophic scar fibroblasts. Zhonghua Shao Shang Za Zhi 32: 594-598, 2016 (In Chinese). 
9. Liu J, Xiao X, Shen Y, Chen L, Xu C, Zhao H, Wu Y, Zhang Q, Zhong J, Tang Z, et al: MicroRNA-32 promotes calcification in vascular smooth muscle cells: Implications as a novel marker for coronary artery calcification. PLoS One 12: e0174138, 2017.

10. Zheng Y, Wang Z and Zhou Z: miRNAs: Novel regulators of autoimmunity-mediated pancreatic $\beta$-cell destruction in type 1 diabetes. Cell Mol Immunol 14: 488-496, 2017.

11. Herter EK and Xu Landén N: Non-coding RNAs: New players in skin wound healing. Adv Wound Care (New Rochelle) 6: 93-107, 2017.

12. Mu S, Kang B, Zeng W, Sun Y and Yang F: MicroRNA-143-3p inhibits hyperplastic scar formation by targeting connective tissue growth factor $\mathrm{CTGF} / \mathrm{CCN} 2$ via the $\mathrm{Akt} / \mathrm{mTOR}$ pathway. Mol Cell Biochem 416: 99-108, 2016.

13. Kim H, Banerjee N, Sirven MA, Minamoto Y, Markel ME, Suchodolski JS, Talcott ST and Mertens-Talcott SU: Pomegranate polyphenolics reduce inflammation and ulceration in intestinal colitis-involvement of the miR-145/p70S6K1/HIF1a axis in vivo and in vitro. J Nutr Biochem 43: 107-115, 2017.

14. Yuan M, Zhang L, You F, Zhou J, Ma Y, Yang F and Tao L: MiR-145-5p regulates hypoxia-induced inflammatory response and apoptosis in cardiomyocytes by targeting CD40. Mol Cell Biochem 431: 123-131, 2017.

15. Faccini J, Ruidavets JB, Cordelier P, Martins F, Maoret JJ, Bongard V, Ferrières $\mathrm{J}$, Roncalli $\mathrm{J}$, Elbaz $\mathrm{M}$ and Vindis $\mathrm{C}$ : Circulating miR-155, miR-145 and let-7c as diagnostic biomarkers of the coronary artery disease. Sci Rep 7: 42916, 2017.

16. Mo D, Yang D, Xiao X, Sun R, Huang L and Xu J: MiRNA-145 suppresses lung adenocarcinoma cell invasion and migration by targeting N-cadherin. Biotechnol Lett 39: 701-710, 2017.

17. Zhu HY, Li C, Zheng Z, Zhou Q, Guan H, Su LL, Han JT, Zhu XX, Wang SY, Li J and Hu DH: Peroxisome proliferator-activated receptor- $\gamma$ (PPAR- $\gamma$ ) agonist inhibits collagen synthesis in human hypertrophic scar fibroblasts by targeting Smad3 via miR-145. Biochem Biophys Res Commun 459: 49-53, 2015.

18. Ahmad J, Baig MA, Ali AA, Al-Huqail A, Ibrahim MM and Qureshi MI: Comparative assessment of four RNA extraction methods and modification to obtain high-quality RNA from Parthenium hysterophorus leaf. 3 Biotech 7: 373, 2017.

19. Livak KJ and Schmittgen TD: Analysis of relative gene expression data using real-time quantitative PCR and the 2(-Delta Delta C(T)) method. Methods 25: 402-408, 2001.

20. Wu J, Zhang D, Li J, Deng X, Liang G, Long Y, He X, Dai T and Ren D: MACC1 induces autophagy to regulate proliferation, apoptosis, migration and invasion of squamous cell carcinoma. Oncol Rep 38: 2369-2377, 2017.

21. Seo GY, Lim Y, Koh D, Huh JS, Hyun C, Kim YM and Cho M: TMF and glycitin act synergistically on keratinocytes and fibroblasts to promote wound healing and anti-scarring activity. Exp Mol Med 49: e302, 2017.

22. Zhang Z, Kuang F, Liu CL, Chen B, Tang WB and Li XJ: Effects of silencing Smad ubiquitination regulatory factor 2 on the function of human hypertrophic scar-derived fibroblasts. Zhonghua Shao Shang Za Zhi 33: 145-151, 2017 (In Chinese).

23. Shen C, Jiang L, Shao H, You C, Zhang G, Ding S, Bian T, Han C and Meng Q: Targeted killing of myofibroblasts by biosurfactant di-rhamnolipid suggests a therapy against scar formation. Sci Rep 6: 37553, 2016.

24. Pfaff N, Liebhaber S, Möbus S, Beh-Pajooh A, Fiedler J, Pfanne A, Schambach A, Thum T, Cantz T and Moritz T: Inhibition of miRNA-212/132 improves the reprogramming of fibroblasts into induced pluripotent stem cells by de-repressing important epigenetic remodelling factors. Stem Cell Res 20: $70-75,2017$.
25. Do DN, Li R, Dudemaine PL and Ibeagha-Awemu EM: MicroRNA roles in signalling during lactation: An insight from differential expression, time course and pathway analyses of deep sequence data. Sci Rep 7: 44605, 2017.

26. Xie HF, Liu YZ, Du R, Wang B, Chen MT, Zhang YY, Deng ZL and Li J: miR-377 induces senescence in human skin fibroblasts by targeting DNA methyltransferase 1. Cell Death Dis 8: e2663, 2017.

27. Zeng X, Huang C, Senavirathna L, Wang P and Liu L: miR-27b inhibits fibroblast activation via targeting TGF $\beta$ signaling pathway. BMC Cell Biol 18: 9, 2017

28. Li Z, Cai J and Cao X: MiR-19 suppresses fibroblast-like synoviocytes cytokine release by targeting toll like receptor 2 in rheumatoid arthritis. Am J Transl Res 8: 5512-5518, 2016.

29. Wang T, O'Brien EC, Rogers JG, Jacoby DL, Chen ME, Testani JM, Bowles DE, Milano CA, Felker GM, Patel CB, et al: Plasma levels of MicroRNA-155 Are Upregulated with long-term left ventricular assist device support. ASAIO J 63: 536-541, 2017.

30. Ohnesorg T, van den Bergen JA, Belluoccio D, Shankara-Narayana N, Kean AM, Vasilaras A, Ewans L, Ayers KL and Sinclair AH: A duplication in a patient with 46,XX ovo-testicular disorder of sex development refines the SOX9 testis-specific regulatory region to $24 \mathrm{~kb}$. Clin Genet 92: 347-349, 2017.

31. Huang CZ, Xu JH, Zhong W, Xia ZS, Wang SY, Cheng D, Li JY, Wu TF, Chen QK and Yu T: Sox9 transcriptionally regulates Wnt signaling in intestinal epithelial stem cells in hypomethylated crypts in the diabetic state. Stem Cell Res Ther 8: 60, 2017.

32. Bremmer F, Behnes CL, Schildhaus HU, Gaisa NT, Reis H, Jarry H, Radzun HJ, Stroebel P and Schweyer S: The role of beta-catenin mutation and SOX9 expression in sex cord-stromal tumours of the testis. Virchows Arch 470: 421-428, 2017.

33. Liu X, Wang H, Zhu Z, Ye Y, Mao H and Zhang S: MicroRNA-105 targets SOX9 and inhibits human glioma cell progression. FEBS Lett 590: 4329-4342, 2016.

34. Li Z, Li B, Niu L and Ge L: miR-592 functions as a tumor suppressor in human non-small cell lung cancer by targeting SOX9. Oncol Rep 37: 297-304, 2017

35. Wang X, Liu Y, Liu X, Yang J, Teng G, Zhang L and Zhou C: MiR-124 inhibits cell proliferation, migration and invasion by directly targeting SOX9 in lung adenocarcinoma. Oncol Rep 35: 3115-3121, 2016.

36. Chang T, Xie J, Li H, Li D, Liu P and Hu Y: MicroRNA-30a promotes extracellular matrix degradation in articular cartilage via downregulation of Sox9. Cell Prolif 49: 207-218, 2016.

37. Li J, Wang L, Liu Z, Zu C, Xing F, Yang P, Yang Y, Dang X and Wang K: MicroRNA-494 inhibits cell proliferation and invasion of chondrosarcoma cells in vivo and in vitro by directly targeting SOX9. Oncotarget 6: 26216-26229, 2015.

This work is licensed under a Creative Commons Attribution-NonCommercial-NoDerivatives 4.0 International (CC BY-NC-ND 4.0) License. 